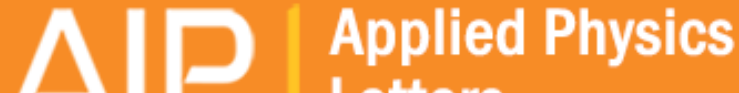 Letters
}

\section{Silver substrates for surface enhanced Raman scattering: Correlation between nanostructure and Raman scattering enhancement}

G. Santoro, S. Yu, M. Schwartzkopf, P. Zhang, Sarathlal Koyiloth Vayalil, J. F. H. Risch, M. A. Rübhausen, M. Hernández, C. Domingo, and S. V. Roth

Citation: Applied Physics Letters 104, 243107 (2014); doi: 10.1063/1.4884423

View online: http://dx.doi.org/10.1063/1.4884423

View Table of Contents: http://scitation.aip.org/content/aip/journal/apl/104/24?ver=pdfcov

Published by the AIP Publishing

\section{Articles you may be interested in}

An ultrasensitive, uniform and large-area surface-enhanced Raman scattering substrate based on $\mathrm{Ag}$ or $\mathrm{Ag} / \mathrm{Au}$ nanoparticles decorated Si nanocone arrays

Appl. Phys. Lett. 106, 043103 (2015); 10.1063/1.4906800

Nanostructured surface enhanced Raman scattering sensor platform with integrated waveguide core

Appl. Phys. Lett. 105, 181101 (2014); 10.1063/1.4900637

"Rings of saturn-like" nanoarrays with high number density of hot spots for surface-enhanced Raman scattering Appl. Phys. Lett. 105, 033515 (2014); 10.1063/1.4891533

One-pot hydrothermal synthesis of silver nanoplates on optical fiber tip for surface-enhanced Raman scattering Appl. Phys. Lett. 104, 201906 (2014); 10.1063/1.4879552

Ag dendritic nanostructures as ultrastable substrates for surface-enhanced Raman scattering

Appl. Phys. Lett. 102, 183118 (2013); 10.1063/1.4803937

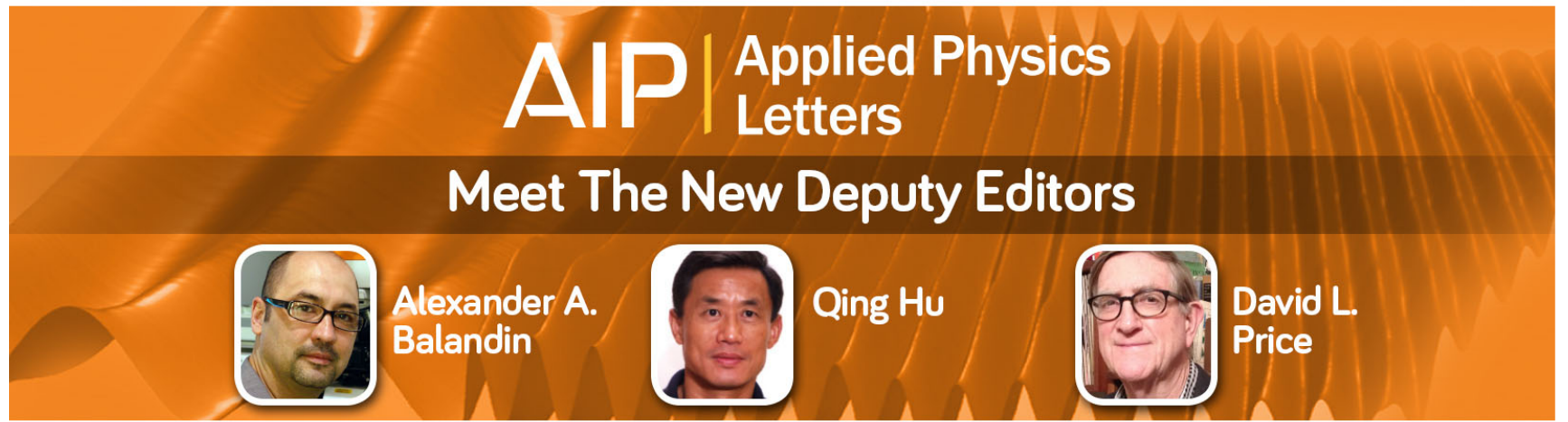




\title{
Silver substrates for surface enhanced Raman scattering: Correlation between nanostructure and Raman scattering enhancement
}

\author{
G. Santoro, ${ }^{1, a)}$ S. Yu, ${ }^{1}$ M. Schwartzkopf, ${ }^{1}$ P. Zhang, ${ }^{1}$ Sarathlal Koyiloth Vayalil, ${ }^{1}$ \\ J. F. H. Risch, ${ }^{1}$ M. A. Rübhausen, ${ }^{2,3}$ M. Hernández, ${ }^{4}$ C. Domingo, ${ }^{4}$ and S. V. Roth ${ }^{1}$ \\ ${ }^{1}$ Photon Science, DESY, Notkestr. 85, D-22607 Hamburg, Germany \\ ${ }^{2}$ Institut für Angewandte Physik, University of Hamburg, Notkestr. 85, D-22607 Hamburg, Germany \\ ${ }^{3}$ Center for Free Electron Laser Science, University of Hamburg, Notkestr. 85, D-22607 Hamburg, Germany \\ ${ }^{4}$ Instituto de Estructura de la Materia, IEM-CSIC, c/Serrano 121-123, E-28006 Madrid, Spain
}

(Received 9 April 2014; accepted 4 June 2014; published online 17 June 2014)

\begin{abstract}
The fabrication of substrates for Surface Enhanced Raman Scattering (SERS) applications matching the needs for high sensitive and reproducible sensors remains a major scientific and technological issue. We correlate the morphological parameters of silver $(\mathrm{Ag})$ nanostructured thin films prepared by sputter deposition on flat silicon ( $\mathrm{Si}$ ) substrates with their SERS activity. A maximum enhancement of the SERS signal has been found at the Ag percolation threshold, leading to the detection of thiophenol, a non-resonant Raman probe, at concentrations as low as $10^{-10} \mathrm{M}$, which corresponds to enhancement factors higher than 7 orders of magnitude. To gain full control over the developed nanostructure, we employed the combination of in-situ time-resolved microfocus Grazing Incidence Small Angle X-ray Scattering with sputter deposition. This enables to achieve a deepened understanding of the different growth regimes of Ag. Thereby an improved tailoring of the thin film nanostructure for SERS applications can be realized. (C 2014 AIP Publishing LLC.
\end{abstract}

[http://dx.doi.org/10.1063/1.4884423]

The tremendous enhancement of the electromagnetic field (EM) in the close vicinity of nanostructured noble metal surfaces is well accepted to constitute the basis of SurfaceEnhanced Raman Scattering (SERS). ${ }^{1}$ This enhancement is primarily due to localized surface plasmons on the metallic nanostructures and the maximum EM enhancement occurs in the gaps between the nanostructures, known as "hot-spots." A chemical enhancement mechanism due to the electronic interaction between the molecule and the metal has also been identified, although this effect is usually smaller than the EM enhancement. $^{2}$

The intensification of the Raman signal achieved through the resonant interaction between the optical fields and localized surface plasmons can lead to an outstanding sensitivity, allowing for the detection of single molecules., Thus, SERS substrates could be used for non-invasive biological assays, ${ }^{5}$ biosensing, ${ }^{6}$ or environmental analysis ${ }^{7}$ with high sensitivity.

Several methods have been commonly employed to fabricate SERS-active substrates, e.g., the immobilization of colloidal nanoparticles on solid surfaces ${ }^{8,9}$ or lithographic techniques. $^{10,11}$ The use of patterned templates exploiting the self-assembly capabilities of block co-polymer thin films has also been explored. ${ }^{12}$ Nevertheless, the fabrication of reliable SERS substrates on macroscopic areas is still an enormous technological challenge.

In order to fabricate homogeneous SERS substrates over macroscopic areas different approaches have recently been investigated such as the laser-direct-writing technique, ${ }^{13}$ the nanosphere lithography method, ${ }^{14}$ or the convective selfassembly of nanoparticles. ${ }^{15}$ However, all of them involve

\footnotetext{
${ }^{\text {a) }}$ Author to whom correspondence should be addressed. Electronic mail: gonzalo.santoro@desy.de
}

several fabrication steps. On the other hand, sputter deposition is a widely employed method in industry that can cover large areas presenting some advantages over wet approaches, ${ }^{16}$ and the preparation of SERS substrates using vacuum deposition is a one-step process that can be applied on a variety of different kinds of substrates with excellent reproducibility. ${ }^{17,18}$ However, to fully exploit its fabrication capabilities for SERS applications, it is of utmost importance to achieve a deep understanding of the growth mechanism of the deposited nanostructured film. In this sense, Grazing Incidence Small Angle X-Ray Scattering (GISAXS) ${ }^{19}$ constitutes a very valuable technique for the morphological characterization of thin films. ${ }^{20,21}$ Recently, it has also been proven to be a very powerful tool for in-situ characterizing the growth of thin nanostructured metallic films, with time resolution in the order of tenths of milliseconds. ${ }^{22-24}$ Due to the shallow incidence angle used, GISAXS provides statistically relevant information over a large sample area.

In this article, we correlate the morphology of nanostructured Ag thin films deposited on flat silicon ( $\mathrm{Si}$ ) substrates and their SERS activity. We have employed radiofrequency (RF) sputter deposition and microfocus GISAXS ( $\mu$ GISAXS) together with SERS measurements at selected film thicknesses. The sensitive, homogeneous over macroscopic areas and easily produced SERS substrates presented here match the needs for reproducible, low-cost sensors based on the SERS effect. In addition, we have followed in-situ and in real-time the morphology development of the deposited thin films, allowing for the extraction of important parameters concerning the growth kinetics and therefore for tuning the nanostructure for specific needs. This information permits to adjust the morphological parameters for further applications such as antimicrobial coatings ${ }^{25}$ or plasmonics. ${ }^{26}$ 
The deposition of Ag (Kurt J. Lesker; Purity 99.99\%) was performed using a portable RF sputter deposition chamber specially designed and manufactured for $\mu$ GISAXS. ${ }^{22}$ Acid-cleaned flat $\mathrm{Si}$ pieces $\left(1.8 \times 1.8 \mathrm{~cm}^{2}\right)$ were used as substrates. ${ }^{27}$ The substrates were kept at room temperature during the entire process and a sputter rate of 0.93 $\pm 0.04 \mathrm{~nm} / \mathrm{min}$ was employed (Ar pressure: $1.8 \times 10^{-2}$ millibars; Power: $100 \mathrm{~W}){ }^{27}$

$\mu$ GISAXS was performed at the P03/MiNaXS beamline ${ }^{28,29}$ of the PETRA III storage ring at DESY (Hamburg, Germany). An incident photon energy of $13 \mathrm{keV}$ with a beam size of $(31 \times 24) \mu \mathrm{m}^{2}$ at the sample position was used. The sample to detector distance was set at $3627 \pm 2 \mathrm{~mm}$ and a PILATUS 1M (Dectris Ltd., Switzerland) with a pixel size of $(172 \times 172) \mu \mathrm{m}^{2}$ was used as detector. During the sputter deposition, the scattering patterns were continuous and uninterruptedly recorded at a frame rate of 10 images per second. In order to achieve a good separation between the $\mathrm{Si}$ and $\mathrm{Ag}$ Yoneda peaks, an incident angle of $\alpha_{i}=0.5^{\circ}$ was used. The $\mu$ GISAXS data were analysed using the DPDAK software package. After performing horizontal and vertical line cuts of the two-dimensional (2D) scattering patterns, the obtained curves were fitted using Lorentzian functions. The simulation of the $\mu$ GISAXS patterns was performed using the IsGISAXS software package. .7,30 $^{27}$

For the SERS characterization, $3 \mu \mathrm{l}$ droplets of thiophenol solution were deposited on the $\mathrm{Ag}$ thin films. The droplets were dried inside a fume hood. A Renishaw InVia Raman microscope (Renishaw plc., United Kingdom) employing an incident laser wavelength of $\lambda=532 \mathrm{~nm}$ and a $50 \times$ objective with a numerical aperture, $\mathrm{NA}=0.75$, leading to a power density of $3.2 \mathrm{~mW} / \mu \mathrm{m}^{2}$ at the sample position, was used for the acquisition of the spectra. Each spectrum was acquired with an acquisition time of $10 \mathrm{~s}$ and 1 accumulation. The enhancement reproducibility of the substrates was tested by acquiring spectra at multiple positions.

To give an overview of the growth process, Fig. 1 presents selected 2D $\mu$ GISAXS patterns obtained during the sputter deposition of Ag. At the early stages of the sputter deposition, two side peaks (along $\mathrm{q}_{\mathrm{y}}$ ) appeared indicating the existence of laterally ordered nanostructures on the sample surface. As the deposition proceeded, the side peaks shifted towards smaller $\mathrm{q}_{\mathrm{y}}$ values, being arrested at a fixed position at later sputtering times. During the entire process both side peaks remained symmetric with respect to the scattering plane. At an effective film thickness around $3 \mathrm{~nm}$ peaks along $\mathrm{q}_{\mathrm{z}}$ became clear. These peaks shifted towards lower $\mathrm{q}_{\mathrm{z}}$ values as the sputter time increased and more modulations appeared and shifted towards lower $\mathrm{q}_{\mathrm{z}}$ indicating the vertical growth of the nanostructured thin film.

In order to extract quantitative information from the scattering patterns, out-of-plane cuts (along $\left.\mathrm{q}_{\mathrm{y}}\right)^{27}$ at the Yoneda peak ${ }^{31}$ position of $\mathrm{Si}\left(\mathrm{q}_{\mathrm{z}, \mathrm{c}}(\mathrm{Si})=0.733 \mathrm{~nm}^{-1}\right)$ and off-detector cuts (along $\left.\mathrm{q}_{\mathrm{z}}\right)^{27}$ at $\mathrm{q}_{\mathrm{y}}=0.112 \mathrm{~nm}^{-1}$ were performed. Figs. 2(a) and 2(b) show 2D maps of the out-ofplane cuts and of the off-detector cuts versus the effective thickness of the deposited film, respectively.

The side peak position as well as the full-width-at-halfmaximum (FWHM) extracted from Lorentzian fittings are depicted in Fig. 2(c). A continuous shift of the side peak position towards lower $\mathrm{q}_{\mathrm{y}}$ values was found indicating an increase in the average distance between $\mathrm{Ag}$ clusters due to coalescence effects. ${ }^{32}$ Furthermore, an overall decrease of the FWHM was observed, revealing an increase in the order of the nanoclusters since the width of the peak is related to the mean cluster distance distribution.

At the early stages of sputter deposition, a change in the slope of the FWHM was identified (inset in Fig. 2(c)), indicating a transition in the growth kinetics from a predominant nucleation process to coalescence of adjacent clusters. ${ }^{23}$ The nucleation threshold was found to occur at an effective film thickness of $\delta=0.28 \pm 0.05 \mathrm{~nm}$. As the deposition continued, the FWHM passed a local maximum at $\delta=5.7 \pm 0.1 \mathrm{~nm}$, revealing a broadening of the cluster distance distribution that can be attributed to the growth of a second layer of Ag. This is in accordance with the observed growth behaviour of ultrathin Ag films deposited from the plasma phase on oxide layers. ${ }^{33,34}$ Below the percolation threshold, a predominantly island growth mechanism occurs whilst a layer-by-layer growth predominates after percolation. $^{32,33}$ Therefore, we identified this point with the percolation threshold of the Ag cluster layer.

From the evolution of the $\mathrm{Si}$ and $\mathrm{Ag}$ Yoneda peaks (Fig. 2(d)) at $\mathrm{q}_{\mathrm{z}, \mathrm{c}}(\mathrm{Si})=0.733 \mathrm{~nm}^{-1}$ and $\mathrm{q}_{\mathrm{z}, \mathrm{c}}(\mathrm{Ag})=0.883 \mathrm{~nm}^{-1}$, respectively, the threshold between two different coalescence mechanisms was identified. At an effective thickness of $\delta=1.1 \pm 0.1 \mathrm{~nm}$, the intensity at the Ag Yoneda peak became dominant, revealing the transition from a coalescence process mainly due to diffusion phenomena to a predominantly adsorption-driven coalescence. ${ }^{23}$ Above the diffusion
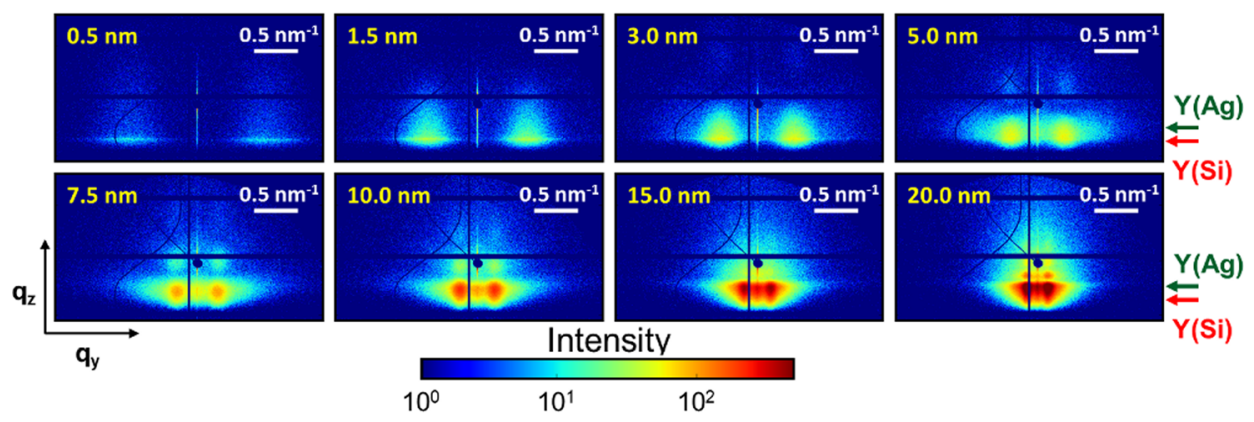

FIG. 1. Selected 2D $\mu$ GISAXS patterns during RF-sputter deposition of Ag. The corresponding effective film thickness is indicated in each image. The dark blue circle corresponds to the specular beam stop used to prevent the detector from being damaged due to the high X-ray intensity. The dark blue horizontal and vertical stripes correspond to the intermodule detector gaps. The positions of the Si Yoneda peak, Y(Si), and Ag Yoneda peak, Y(Ag), as well as the coordinate system $\left(\mathrm{q}_{\mathrm{y}}, \mathrm{q}_{\mathrm{z}}\right)$ are indicated. 

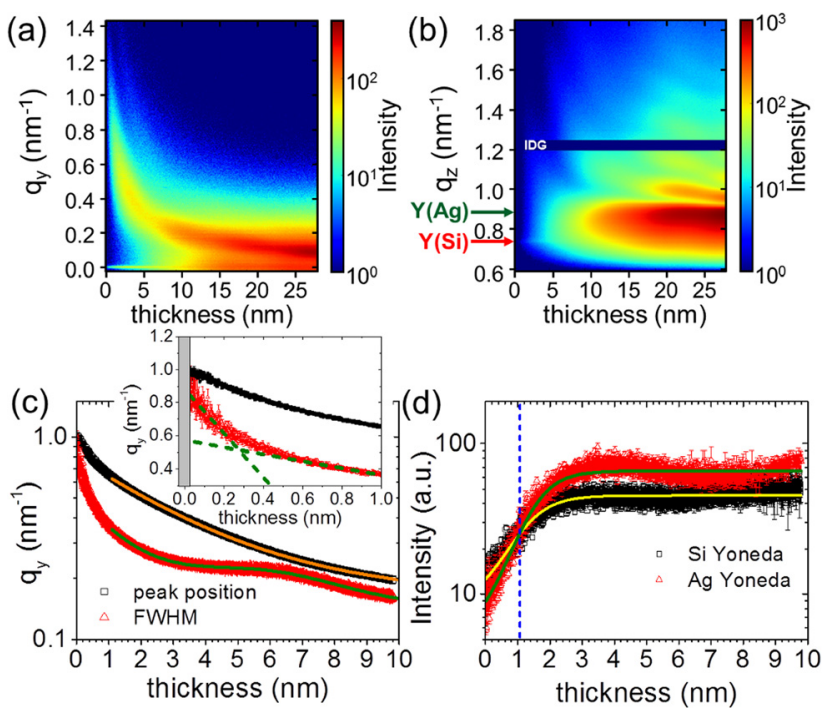

FIG. 2. (a) Out-of-plane (along $\mathrm{q}_{\mathrm{y}}$ ) line cuts versus sputtered thickness at the Si Yoneda peak $\left(\mathrm{q}_{\mathrm{z}, \mathrm{c}}(\mathrm{Si})=0.733 \mathrm{~nm}^{-1}\right)$. (b) Off-detector $\left(\right.$ along $\mathrm{q}_{\mathrm{z}}$ ) line cuts versus sputtered thickness at $\mathrm{q}_{\mathrm{y}}=0.112 \mathrm{~nm}^{-1}$. IDG: Intermodule Detector Gap. Y(Si): Si Yoneda peak. Y(Ag): Ag Yoneda peak. (c) Evolution of the peak position and FWHM of the side peak. The continuous orange line is the exponential fitting of the peak position and the continuous green line represents an exponential plus Gaussian fitting of the FWHM. The inset shows a zoom of the early stage of $\mathrm{Ag}$ deposition. (d) $\mathrm{Si}$ and $\mathrm{Ag}$ Yoneda peak intensities versus the effective sputtered thickness. The continuous lines are Boltzmann sigmoidal fittings of the data. The blue dashed line marks the crossing point of the sigmoidal fittings.

threshold, the shift in the side peak position is well described by an exponential decay $\mathrm{q}_{\mathrm{y}}(\delta)=\mathrm{q}_{0}+\mathrm{A} \exp \left(-\delta / \delta_{0}\right)$, where $\mathrm{q}_{0}=0.16 \mathrm{~nm}^{-1}, \mathrm{~A}=0.64 \mathrm{~nm}^{-1}$, and $\delta_{0}=3.36 \mathrm{~nm}$ (continuous line in Fig. 2(c)). This permits to extract the mean cluster distance throughout the sputter deposition process using the relation $D \approx 2 \pi / \mathrm{q}_{\mathrm{y}}$.

The homogeneity of the deposited film was investigated by performing a lateral $\mu$ GISAXS scan after the complete deposition. No variation in the scattering patterns was found indicating that the nanostructured thin film was homogeneous over the scanned area. ${ }^{27}$ Furthermore, no influence of the $\mathrm{X}$-ray beam on the Ag growth was observed. ${ }^{27}$

To verify the SERS activity of the substrates, several droplets of a $10^{-4} \mathrm{M}$ thiophenol solution were deposited on $\mathrm{Ag}$ layers with different effective thickness and nanostructure deduced from the in-situ $\mu$ GISAXS study. We also deposited a droplet on a bare Si substrate for comparison. Thiophenol was employed as Raman probe instead of the more commonly used Rhodamine 6G and crystal violet since the latter ones exhibit Raman resonance effects under visible illumination, obscuring the SERS enhancement solely due to surface effects. Whilst no thiophenol signal was observed on
$\mathrm{Si}$, the characteristic SERS bands of thiophenol adsorbed on $\mathrm{Ag}^{35}$ became clear for all the substrates (Fig. 3(a)).

The Raman intensity was quantified using the integrated peak area of the $999 \mathrm{~cm}^{-1}, 1023 \mathrm{~cm}^{-1}$, and $1074 \mathrm{~cm}^{-1}$ bands, corresponding to the benzene ring breathing mode, the in-plane $\mathrm{CH}$ bending mode and a combination of the benzene ring breathing and CS stretching modes, respectively. ${ }^{36}$ A maximum intensification of the SERS signal was found for an effective film thickness of $5.6 \pm 0.1 \mathrm{~nm}$ (Fig. 3(b)), which corresponds to the percolation threshold identified by the in-situ $\mu$ GISAXS study. Below and above the percolation threshold, the SERS enhancement was found to decrease symmetrically. As the nanostructured film grows, the gap between clusters is reduced, and a higher intensification of the local electromagnetic field takes place. However, when the percolation threshold is passed, the film presents a high connectivity, and thus, the density of available "hot-spots" is significantly reduced. At the percolation threshold, the optical properties of the film changes drastically, and the maximum surface plasmon resonance takes place. ${ }^{33,37}$

Fig. 3(c) shows the integrated intensity of the aforementioned SERS bands at different thiophenol concentrations for the substrate with an effective thickness of $5.6 \pm 0.1 \mathrm{~nm}$. As the thiophenol solution was diluted, a decrease of the SERS intensity was observed up to a concentration of $10^{-7} \mathrm{M}$ followed by a saturation of the Raman enhancement. This behaviour is found for molecules that adsorb to the surface of SERS substrates. ${ }^{38}$ The SERS spectrum of thiophenol was still visible for a concentration as low as $10^{-10} \mathrm{M}$, and from the saturation effect, the SERS enhancement factor can be estimated to be higher than 7 orders of magnitude. This estimation is in good agreement with the calculated enhancement factors for several different SERS bands of thiophenol. ${ }^{27}$

In order to extract relevant morphological information concerning the observed SERS activity of the substrates, we simulated the $\mu \mathrm{GISAXS}$ patterns assuming a hexagonal paracrystalline arrangement of hemispherical Ag nanoclusters. ${ }^{23,34}$ We also assumed that all the material deposited is distributed into clusters, i.e., the total volume of the clusters equals the volume of a homogenous layer with the corresponding effective thickness. The position and shape of the side peak (Fig. 4, middle row) show an excellent agreement with the experimental data (Fig. 4, upper row), confirming the hemispherical shape of the clusters for all thicknesses. Therefore, the mean radii and mean gap between $\mathrm{Ag}$ clusters have been extracted. The results are presented in Table I and depicted as sketches in the lower row of Fig. 4.

For the substrate presenting the maximum SERS enhancement $(\delta=5.6 \pm 0.1 \mathrm{~nm})$ a mean gap of around $1 \mathrm{~nm}$ was calculated. In the model we have used, effective film
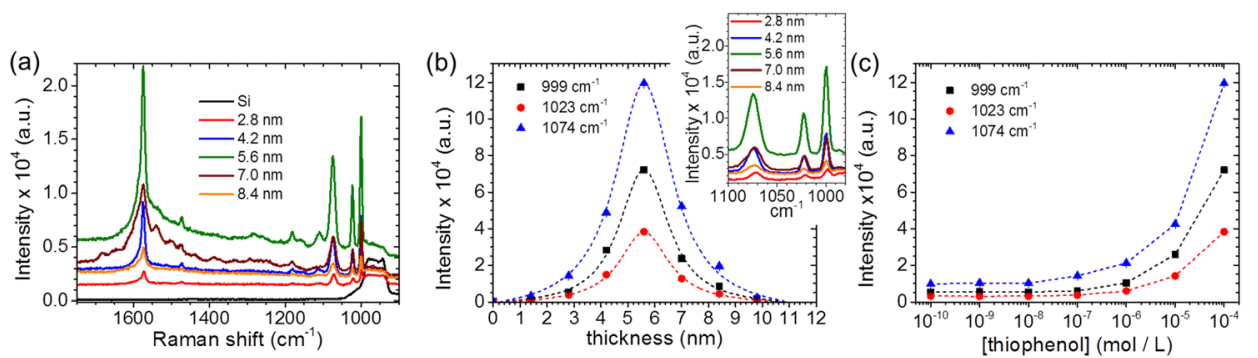

FIG. 3. (a) Surface-Enhanced Raman spectra of thiophenol on sputtered $\mathrm{Ag}$ samples at different film thicknesses. (b) Intensity of the characteristic SERS bands shown in the inset for different $\mathrm{Ag}$ thickness. The dashed lines are Voigt fittings to the data. (c) Intensity of the characteristic SERS bands at different thiophenol concentrations on the $5.6 \mathrm{~nm} \mathrm{Ag}$ thickness film. 
$2.8 \mathrm{~nm}$

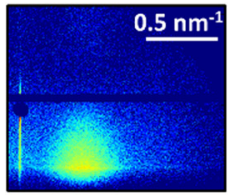

$0.5 \mathrm{~nm}^{-1}$
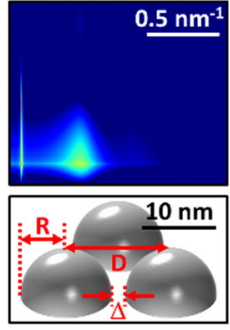

$4.2 \mathrm{~nm}$

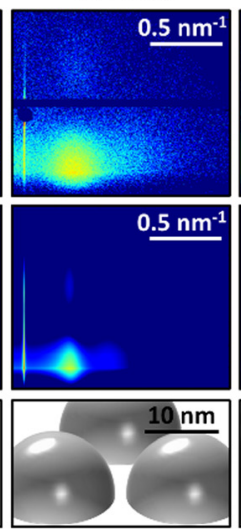

$5.6 \mathrm{~nm}$

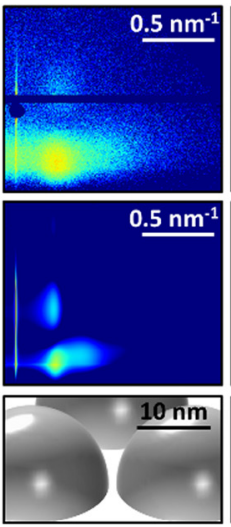

$7.0 \mathrm{~nm}$

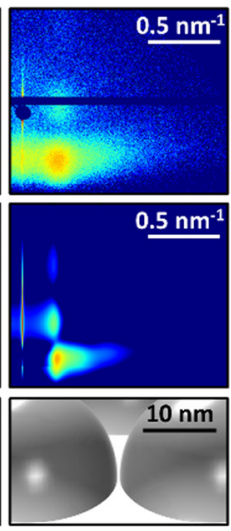

$8.4 \mathrm{~nm}$

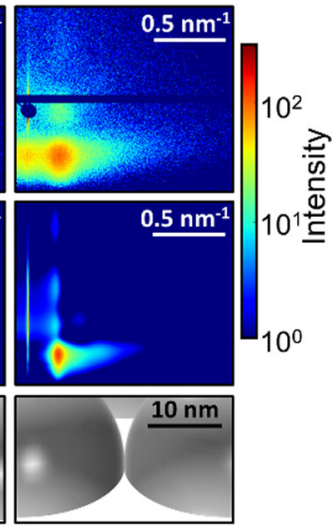

FIG. 4. Upper row: 2D- $\mu$ GISAXS patterns for the thicknesses at which the SERS activity of the Ag thin films was investigated. Middle row: Simulated 2D- $\mu$ GISAXS patterns. Lower row: Real space sketches of the morphology. $\mathrm{D}=$ mean cluster correlation distance; $\mathrm{R}=$ mean cluster radius; and $\Delta=$ mean gap between clusters.
TABLE I. Morphological parameters of the nanostructured Ag thin films extracted from the $\mu$ GISAXS data simulation.

\begin{tabular}{cccc}
\hline \hline$\delta^{\mathrm{a}}(\mathrm{nm})$ & $\mathrm{D}^{\mathrm{b}}(\mathrm{nm})$ & $\mathrm{R}^{\mathrm{c}}(\mathrm{nm})$ & $\Delta^{\mathrm{d}}(\mathrm{nm})$ \\
\hline $2.8 \pm 0.1$ & $14.2 \pm 0.1$ & $6.3 \pm 0.1$ & $1.6 \pm 0.1$ \\
$4.2 \pm 0.1$ & $18.1 \pm 0.1$ & $8.4 \pm 0.1$ & $1.3 \pm 0.1$ \\
$5.6 \pm 0.1$ & $21.9 \pm 0.1$ & $10.5 \pm 0.1$ & $0.9 \pm 0.1$ \\
$7.0 \pm 0.1$ & $25.8 \pm 0.1$ & $12.7 \pm 0.1$ & $0.4 \pm 0.1$ \\
$8.4 \pm 0.1$ & $28.8 \pm 0.1$ & $14.4 \pm 0.1$ & $0.0 \pm 0.1$ \\
\hline \hline
\end{tabular}

${ }^{\mathrm{a}}$ Effective film thickness.

${ }^{\mathrm{b}}$ Mean cluster correlation distance.

${ }^{\mathrm{c}}$ Mean cluster radius.

${ }^{\mathrm{d}}$ Mean gap between clusters.

thicknesses above $5.6 \mathrm{~nm}$ have smaller mean gaps, what could lead to higher SERS enhancements. ${ }^{39}$ Nevertheless, the high connectivity of the deposited clusters and the increased electron tunnelling probability between nanostructures $^{40}$ are responsible for the decrease of the observed SERS enhancement.

In summary, SERS substrates over macroscopic areas have been prepared by RF-sputter deposition of Ag finding enhancement factors higher than 7 orders of magnitude for an effective film thickness corresponding to the percolation threshold, extracted from the in-situ $\mu$ GISAXS measurements. At this effective thickness a mean gap between hemispherical $\mathrm{Ag}$ clusters of around $1 \mathrm{~nm}$ has been found. Moreover, thiophenol was still detected at concentrations as low as $10^{-10} \mathrm{M}$. The substrates presented homogeneous nanostructured morphology over macroscopic areas and exhibited high SERS sensitivity which, together with the easiness of fabrication, are the specific needs for SERSbased sensors. In addition, from the unique combination of in-situ time resolved $\mu$ GISAXS and sputter deposition, we have identified the main growth kinetic regimes as well as their thresholds, which is crucial for nanostructure tailoring. This information is of high relevance not only for the purpose of this article but also for adjusting the nanostructured Ag morphology to further different specific requirements.

Parts of this research were carried out at the light source PETRA III at DESY, a member of the Helmholtz Association (HGF). The authors acknowledge DESY for beam time granted in the frame of the Project No. I-20120504 EC. The authors kindly acknowledge Ralph Döhrmann for the help during the in-situ sputter deposition $\mu$ GISAXS measurements. They would also like to thank Marián Gómez-Fatou and David Gómez for the FE-SEM measurements. S.Y. acknowledges the Knut och Alice Wallenberg foundation for the kind financial support. M.A.R. acknowledges the Deutsche Forschungsgemeinschaft for funding under the Project No. FOR 1405.

${ }^{1}$ M. Moskovits, J. Raman Spectrosc. 36, 485 (2005).

${ }^{2}$ K. Kneipp, Phys. Today 60(11), 40 (2007).

${ }^{3}$ S. M. Nie and S. R. Emery, Science 275, 1102 (1997).

${ }^{4}$ K. Kneipp, Y. Wang, H. Kneipp, L. T. Perelman, I. Itzkan, R. Dasari, and M. S. Feld, Phys. Rev. Lett. 78, 1667 (1997).

${ }^{5}$ S. Feng, J. Lin, Z. Huang, G. Chen, W. Chen, Y. Wang, R. Chen, and H. Zeng, Appl. Phys. Lett. 102, 043702 (2013).

${ }^{6}$ J. Chen, G. W. Qin, J. S. Wang, J. Y. Yu, B. Shen, S. Li, Y. P. Ren, L. Zuo, W. Shen, and B. Das, Biosens. Bioelectron. 44, 191 (2013).

${ }^{7}$ L. Guerrini, J. V. Garcia-Ramos, C. Domingo, and S. Sanchez-Cortes, Anal. Chem. 81, 1418 (2009).

${ }^{8}$ M. V. Cañamares, J. V. Garcia-Ramos, J. D. Gomez-Varga, C. Domingo, and S. Sanchez-Cortes, Langmuir 21, 8546 (2005).

${ }^{9}$ M. R. Barmi, C. Andreou, M. R. Hoonejani, M. Moskovits, and C. D. Meinhart, Langmuir 29, 13614 (2013).

${ }^{10}$ A. Gopinath, S. V. Boriskina, W. R. Premasiri, L. Ziegler, B. M. Reinhard, and L. Dal Negro, Nano Lett. 9, 3922 (2009).

${ }^{11}$ Q. Fu, D. Zhang, Y. Chen, X. Wang, L. Han, L. Zhu, P. Wang, and H. Ming, Appl. Phys. Lett. 103, 041122 (2013).

${ }^{12}$ F. L. Yap, P. Thoniyot, S. Krishnan, and S. Krishnamoorthy, ACS Nano 6, 2056 (2012).

${ }^{13}$ M. L. Tseng, Y. W. Huang, M. K. Hsiao, H. W. Huang, H. M. Chen, Y. L. Chen, C. H. Chu, N. N. Chu, Y. J. He, C. M. Chang et al., ACS Nano 6, 5190 (2012).

${ }^{14}$ Z. Dai, X. Xiao, L. Liao, J. Zheng, F. Mei, W. Wu, J. Ying, F. Ren, and C. Jiang, Appl. Phys. Lett. 103, 041903 (2013).

${ }^{15}$ C. Farcau, N. M. Sangeetha, N. Decorde, S. Astilean, and L. Ressier, Nanoscale 4, 7870 (2012).

${ }^{16}$ F. Faupel, V. Zaporojtchenko, H. Greve, U. Schürmann, V. S. K. Chakravadhanula, C. Hanisch, A. Kulkarni, A. Gerber, E. Quandt, and R. Podschun, Contrib. Plasma Phys. 47, 537 (2007).

${ }^{17}$ J. D. Driskell, S. Shanmukh, Y. Liu, S. B. Chaney, X. J. Tang, Y. P. Zhao, and R. A. Dluhy, J. Phys. Chem. C 112, 895 (2008).

${ }^{18}$ J. P. Singh, H. Chu, J. Abell, R. A. Tripp, and Y. Zhao, Nanoscale 4, 3410 (2012).

${ }^{19}$ P. Müller-Buschbaum, Anal. Bioanal. Chem. 376, 3 (2003).

${ }^{20}$ S. V. Roth, M. Burghammer, C. Riekel, P. Müller-Buschbaum, A. Diehert, P. Panagiotou, and H. Walter, Appl. Phys. Lett. 82, 1935 (2003).

${ }^{21}$ D. Babonneau, S. Camelio, E. Vandenhecke, S. Rousselet, M. Garel, F. Pailloux, and P. Boesecke, Phy. Rev. B 85, 235415 (2012).

${ }^{22}$ R. Döhrmann, S. Botta, A. Buffet, G. Santoro, K. Schlage, M. Schwartzkopf, S. Bommel, J. F. H. Risch, R. Mannweiler, S. Brunner et al., Rev. Sci. Instrum. 84, 043901 (2013).

${ }^{23}$ M. Schwartzkopf, A. Buffet, V. Körstgens, E. Metwalli, K. Schlage, G. Benecke, J. Perlich, M. Rawolle, A. Rothkirch, B. Heidmann et al., Nanoscale 5, 5053 (2013). 
${ }^{24}$ S. Yu, G. Santoro, K. Sarkar, B. Dicke, P. Wessels, S. Bommel, R. Döhrmann, J. Perlich, M. Kuhlmann, E. Metwalli et al., J. Phys. Chem. Lett. 4, 3170 (2013).

${ }^{25}$ J. S. Kim, E. Kuk, K. N. Yu, J. H. Kim, S. J. Park, H. J. Lee, S. H. Kim, Y. K. Park, Y. H. Park, C. Y. Hwang et al., Nanomed. Nanotechnol. Biol. Med. 3, 95 (2007).

${ }^{26}$ L. González-García, J. Parra-Barranco, J. R. Sánchez-Valencia, J. Ferrer, M. C. García-Gutiérrez, A. Barranco, and A. R. González-Elipe, Adv. Funct. Mater. 23, 1655 (2013).

${ }^{27}$ See supplementary material at http://dx.doi.org/10.1063/1.4884423 for the description of the principle of $\mu$ GISAXS, substrate pre-treatment, sputter deposition parameters and rate (Spectroscopic ellipsometry, FE-SEM), $\mu$ GISAXS lateral scan after deposition, SERS enhancement factor calculations, details of $\mu$ GISAXS data simulation and Field-Emission Scanning Electron Microscopy.

${ }^{28}$ A. Buffet, A. Rothkirch, R. Dohrmann, V. Körstgens, M. M. A. Kashem, J. Perlich, G. Herzog, M. Schwartzkopf, R. Gehrke, P. Müller-Buschbaum et al., J. Synchrotron. Radiat. 19, 647 (2012).
${ }^{29}$ G. Santoro, A. Buffet, R. Döhrmann, S. Yu, V. Körstgens, P. MüllerBuschbaum, U. Gedde, M. Hedenqvist, and S. V. Roth, Rev. Sci. Instrum. 85, 043901 (2014).

${ }^{30}$ R. Lazzari, J. Appl. Crystallogr. 35, 406 (2002).

${ }^{31}$ Y. Yoneda, Phys. Rev. 131, 2010 (1963).

${ }^{32}$ J. S. Maa, J. I. Lee, and T. E. Hutchins, J. Vac. Sci. Technol. 11, 136 (1974).

${ }^{33}$ T. W. H. Oates and A. Mucklich, Nanotechnology 16, 2606 (2005).

${ }^{34}$ S. Grachev, M. de Grazia, E. Barthel, E. Sondergard, and R. Lazzari, J. Phys. D: Appl. Phys. 46, 375305 (2013).

${ }^{35}$ K. T. Carron and L. G. Hurley, J. Phys. Chem. 95, 9979 (1991).

${ }^{36}$ T. H. Joo, M. S. Kim, and K. Kim, J. Raman Spectrosc. 18, 57 (1987).

${ }^{37}$ E. M. Akinoglu, T. Y. Sun, J. W. Gao, M. Giersig, Z. F. Ren, and K. Kempa, Appl. Phys. Lett. 103, 117106 (2013).

${ }^{38}$ I. Izquierdo-Lorenzo, J. V. Garcia-Ramos, and S. Sanchez-Cortes, J. Raman Spectrosc. 44, 1422 (2013).

${ }^{39}$ E. Hao and G. C. Schatz, J. Chem. Phys. 120, 357 (2004).

${ }^{40}$ A. Otto, J. Raman Spectrosc. 33, 593 (2002). 